\title{
Effect of liquid storage on membrane integrity and mitochondrial activity: a new diagnostic method of evaluating boar sperm quality*
}

\author{
M. Trzcińska ${ }^{1}$, M. Bryła and Z. Smorąg \\ National Research Institute of Animal Production, \\ Department of Biotechnology of Animal Reproduction \\ 32-083 Balice, Poland
}

(Received 10 March 2008; revised version 30 April 2008; accepted 24 June 2008)

\begin{abstract}
The aim of this study was to determine sperm membrane integrity and mitochondrial activity, to quantify possible apoptosis, and to investigate relationship between these parameters. Apoptotic-like changes were evaluated using a combination of YO-PRO-1/PI staining, and changes in mitochondrial membrane potential were evaluated using JC- 1 . Gel-free ejaculates collected from five boars were extended in BTS diluent and stored for six days at $15-17^{\circ} \mathrm{C}$. The fluorescence analysis was repeated on the first, third and sixth day of semen storage. Motility was also assessed on each day of the experiment. The percentage of apoptotic sperm detected by YO-PRO-1/PI staining inconsiderable decreased, while number of necrotic sperm increased during six days storage. The percentage of spermatozoa with mitochondrial membrane potential $(\Delta \Psi m)$ dysfunction increased and reached the highest level at day six of semen storage. The $\Delta \Psi m$ analysis by JC-1 revealed that mitochondrial function was highly correlated with both sperm motility $(\mathrm{r}=0.936)$ and apoptosis/necrosis evaluated by the YO-PRO-1/PI assay $(r=0.960)$.
\end{abstract}

KEY WORDS: boar spermatozoa, membrane integrity, mitochondrial activity, apoptotisis

\section{INTRODUCTION}

The quality of sperm samples is one of the factors that determine successful insemination. More than $99 \%$ of the estimated 19 million inseminations of gilts conducted world-wide use extended semen in a liquid state, which were stored at

\footnotetext{
* Supported by the State Committee for Scientific Research, Grant No. 2P06D 02330

${ }^{1}$ Corresponding author: e-mail: mcala@izoo.krakow.pl
} 
$15-20^{\circ} \mathrm{C}$ for several days until used for artificial insemination (Huo et al., 2002). Currently, sperm quality is evaluated by conventional semen analysis, which determines sperm concentration and motility. Although the conventional analysis of semen yields considerable information, new methods are still needed to make the evaluation of sperm more reliable. Recent studies have demonstrated that detection of apoptotic and necrotic changes in ejaculated sperm may be a new criterion for evaluating semen quality (Chen et al., 2006).

Apoptosis is a carefully regulated process of cell death that occurs as a normal part of development. It is a well characterized mechanism that allows eukaryotes to eliminate unneeded, senescent, or aberrant cells. While apoptosis in somatic cells as well as spermatocytes and spermatides in vivo is well established, the presence and significance of apoptosis in ejaculated animal sperm is still unresolved (Oehninger et al., 2003). Normal spermatogenesis depends on the efficiency of apoptosis. Approximately $25-75 \%$ of germ cells degenerate in adult mammalian testis. Apoptosis has been clearly observed in rat testis, affecting spermatogonia, spermatocytes and spermatides (Lue et al., 1999). Thus, apoptotic cell populations in ejaculated sperm might result from abortive apoptosis of sperm that avoided elimination mechanisms during spermatogenesis (Sakkas et al., 1999, 2004).

Some apoptotic-like phenomena have been suggested to occur in mature spermatozoa, such as the translocation of phosphatidylserine (PS) from an inner to an outer leaflet of sperm membrane (Pena et al., 2005). Research carried out to date has shown that the presence of apoptotic spermatozoa in fresh semen could be one of the reasons for poor fertility in breeding bulls (Anzar et al., 2002), and appears to be a cause of male infertility in humans (Marchetti et al., 2002). Moreover, semen from infertile men contains a high proportion of spermatozoa with typical signs of apoptosis, including morphological damage, nuclear DNA fragmentation, and loss of asymmetric distribution of PS at the plasma membrane (Barroso et al., 2000). During apoptosis the cytoplasmic membrane becomes slightly permeant. Certain dyes, such as the green fluorescent YO-PRO-1 dye can enter apoptotic cells, whereas other dyes, such as the red fluorescent dye, propidium iodide (PI), cannot. Thus, use of YO-PRO-1 dye and PI together provide a sensitive indicator for apoptosis. The Vybrant Apoptosis Assay Kit \#4 provides assay for apoptosis.

A number of crucial events in apoptosis originate in mitochondria. These organelles are required for efficient energy metabolism, production of membrane lipids, and cell growth. They are also primary determinants of cellular life or death. Following the induction of apoptosis, mitochondrial pores are opened, resulting in a decrease of mitochondrial membrane potential $(\Delta \Psi m)$. Mitochondria have been verified as coordinators of apoptosis in numerous cell systems (Donnelly et al., 2000). Knowledge of the amount of viable spermatozoa that have a stable plasma membrane and have high mitochondrial potential would give very valuable 
information about their quality. The lipophilic cationic fluorescent carbocyanine dye, JC-1, is used to differentially label mitochondria with high and low membrane potential. JC-1 monomers emit a green fluorescence in mitochondria with low potential, while emitting a bright red-orange fluorescence in case of multimer formation, known J-aggreggates, in mitochondria with high membrane potential.

The aim of the current study was to determine the boar sperm membrane integrity, mitochondrial activity, and motility following storage at $15-17^{\circ} \mathrm{C}$. We used both the Vybrant Apoptosis Assay Kit and JC-1 probe, and also asked if any correlation exists between mitochondrial function, sperm motility and apoptosis/ necrosis changes.

\section{MATERIAL AND METHODS}

\section{Semen collection and preparation}

Five crossbred boars aged less than two years were used in this study. Whole ejaculates were collected by hand manipulation into water-jacketed vessels. Three ejaculates were obtained from each boar. After separation from the gel, the semen was filtered through gauze. Sperm volume, concentration, and motility were estimated immediately after collection. Semen samples were diluted to a final concentration of $30 \times 10^{6} \mathrm{sperm} / \mathrm{ml}$ in BTS (Beltsville Thawing Solution, IMV, L'Aigle, France) extender, after which they were placed in cold storage at 15$17^{\circ} \mathrm{C}$ for six days.

\section{Microscopic examinations of sperm motility}

Motility was assessed on each day of experiment, i.e. on days 1, 3 and 6 of semen storage. Samples of semen were incubated for $30 \mathrm{~min}$. The percentage of motile spermatozoa was then evaluated under a phase-contrast optical microscope $\left(200 \times\right.$ magnification) equipped with a heated plate at $37^{\circ} \mathrm{C}$.

\section{Fluorescent staining to assess sperm viability using YO-PRO-1/PI assay}

Fluorescence analysis was performed on the first, third and sixth day of semen storage. The Vybrant Apoptosis Assay Kit \#4 (Molecular Probes Inc., Eugene, USA) was used to detect changes in plasma membrane permeability to YO-PRO-1 related to apoptosis (Idziorek et al., 1995; Martin et al., 2004). A total of $2 \times 10^{6}$ sperm cells were diluted in $1 \mathrm{ml}$ phosphate buffered saline (PBS, Sigma-Aldrich Chemie Gmbh, Steinheim, Germany), and $1 \mu 1$ of YO-PRO-1 (100 $\mu \mathrm{mol} / 1)$ was 
added. The tubes were gently mixed and incubated for $20 \mathrm{~min}$ at room temperature after which $2 \mu \mathrm{mol} / 1 \mathrm{PI}$ was added to each tube. After the incubation period, the sperm cell suspensions were analysed under a fluorescence microscope at 400 $\times$ magnification. At least 200 spermatozoa per sample were evaluated using appropriate filters for red (PI) and green (YO-PRO-1) fluorescence detection.

\section{Fluorescent examinations of mitochondrial function using JC-1 probe}

For evaluation of the mitochondrial membrane potential, spermatozoa were labeled with JC-1 (Molecular Probes Inc., Eugene, USA). Semen was centrifuged for $15 \mathrm{~min}$ at $300 \mathrm{~g}$ at room temperature and the pellet of sperm was then washed with PBS (without calcium and magnesium, Sigma-Aldrich Chemie Gmbh, Steinheim, Germany). Finally, the sample was centrifuged and washed twice, and then the sperm pellet was resuspended in $1 \mathrm{ml}$ PBS. The sample was stained with $10 \mu \mathrm{g} / \mathrm{ml} \mathrm{JC}-1$ (final concentration; stock solution $1 \mathrm{mg} / \mathrm{ml}$ in DMSO) at $37^{\circ} \mathrm{C}$ for $15 \mathrm{~min}$. After incubation, sperm smears were evaluated with a fluorescence microscope (Nikon Eclipse E600, Tokyo, Japan). At least 200 spermatozoa per sample were evaluated using appropriate filters.

\section{Statistical analysis}

Results are expressed as means \pm SEM. Data were analysed by the variance method according to the GLM procedure of the Statistical Analysis System (SAS). Significance of differences between means was tested at $\mathrm{P}<0.01$ by Chisquare test. The correlations between fluorescence parameters and motility were performed using Pearson's method.

\section{RESULTS}

A decrease in motility was observed during semen storage. There was a reduction $(\mathrm{P}<0.01)$ of percentage of motile spermatozoa from day $1(80 \pm 6 \%)$ to day $6(44 \pm 17 \%)$ (Figure 1).

Using this combination of fluorophores, three sperm subpopulations were observed under fluorescence microscopy (Table 1). The first subpopulation, in which no fluorescence was observed, was live sperm with intact membranes (YOPRO-1 $\left./ \mathrm{PI}^{-}\right)$. The second sperm subpopulation (YO-PRO-1 $1^{+} / \mathrm{PI}^{-}$) was apoptotic sperm, showing only green fluorescence. The third subpopulation consisted of necrotic spermatozoa stained with YO-PRO-1 and PI (YO-PRO- $1^{+} / \mathrm{PI}^{+}$), which fluoresced green and red on the fluorescence microscope (Figure 2). 


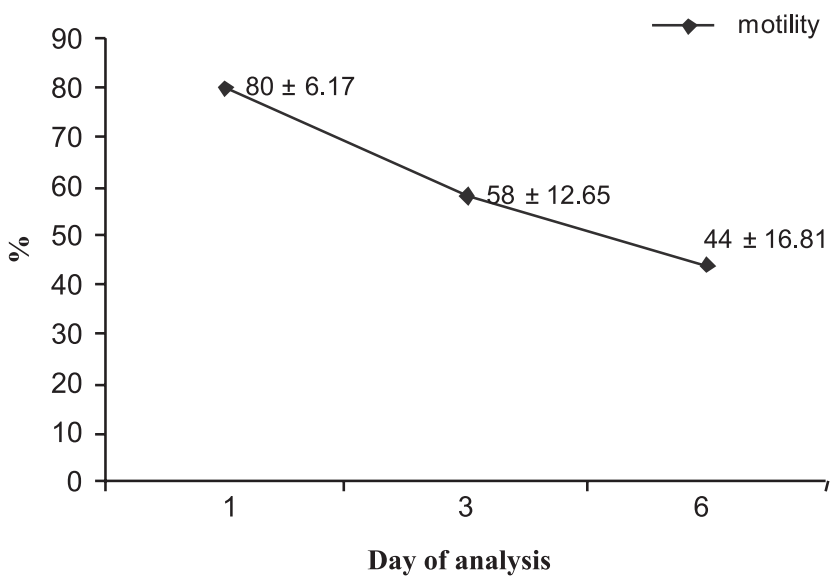

Figure 1. Average percentage of motility in 5 boars ( 3 samples/boar)

Decreasing of live and apoptotic sperm was observed from day $1(80.5 \pm 5.8 \%$; $2.7 \pm 11 \%)$ to day $6(51.0 \pm 17.9 \% ; 1.6 \pm 2.4 \%)$, respectively. There was an increasing of percentage of necrotic sperm from day $1(16.7 \pm 5.7 \%)$ to day $6(51.0 \pm 17.3 \%)$ (Table 1).

Table 1. Percentage (mean $\pm \mathrm{SD}$ ) of apoptotic, necrotic and live sperm stained with YO-PRO-1/PI in 5 boars ( 3 samples/boar)

\begin{tabular}{cccc}
\hline $\begin{array}{l}\text { Day of sperm } \\
\text { storage }\end{array}$ & $\begin{array}{c}\text { Live sperm } \\
\left(\text { YO-PRO- }^{-/ P I}\right)\end{array}$ & $\begin{array}{c}\text { Apoptotic sperm } \\
\left(\text { YO-PRO- }^{+} / \mathrm{PI}^{-}\right)\end{array}$ & $\begin{array}{c}\text { Necrotic sperm } \\
\text { YO-PRO-1 }^{+} / \mathrm{PI}^{+}\end{array}$ \\
\hline 1 & $80.5 \pm 5.8$ & $2.7 \pm 1.1$ & $16.7 \pm 5.7$ \\
3 & $66.3 \pm 10.6$ & $4.0 \pm 1.7$ & $31.5 \pm 10.6$ \\
6 & $51.0 \pm 17.9$ & $1.6 \pm 2.4$ & $51.0 \pm 17.3$ \\
\hline
\end{tabular}

Images represent live sperm with no fluorescence (YO-PRO-1-/PI'), apoptotic spermatozoa (YO-PRO-1 $\left.1^{+} / \mathrm{PI}^{-}\right)$, showing only green fluorescence ( $\left.\mathrm{A}^{\prime}, \mathrm{A}^{\prime \prime}\right)$, and necrotic spermatozoa (YO-PRO- $1^{+} / \mathrm{PI}^{+}$) stained both with YO-PRO-1 and PI (YO-PRO-1'/PI; B', B").

Percentage of spermatozoa with high and low mitochondrial potential assessed by JC- 1 staining is shown in Table 2 . In live sperm, JC- 1 is taken up by the mitochondria, where it forms aggregates that exhibit red/orange fluorescence. In dysfunctional sperm (possibly apoptotic/necrotic), JC-1 cannot aggregate in the mitochondria due to alterations in membrane potential. The stain remains as a monomer in the cytoplasm where it fluoresces green (Figure 3). The increase in $\Delta \Psi m$ causes a fluorescence emission shift from green to red-orange due to the reversible formation of JC-1 aggregates in polarized mitochondria. There were $82.6 \pm 3.5 \%$ of spermatozoa with high $\Delta \Psi m$ on first day of analysis, which decreased with storage time to $51.2 \pm 17.2 \%$. 
Table 2. Percentage (mean $\pm \mathrm{SD}$ ) of mitochondrial potential determined by JC-1 in 5 boars (3 samples/boar)

\begin{tabular}{ccc}
\hline Day of analysis & Sperm with high $\Delta \Psi m$ & Sperm with low $\Delta \Psi m$ \\
\hline 1 & $82.6 \pm 3.5$ & $17.8 \pm 3.3$ \\
3 & $65.7 \pm 13.2$ & $34.3 \pm 13.2$ \\
6 & $51.2 \pm 17.2$ & $48.8 \pm 17.2$ \\
\hline
\end{tabular}
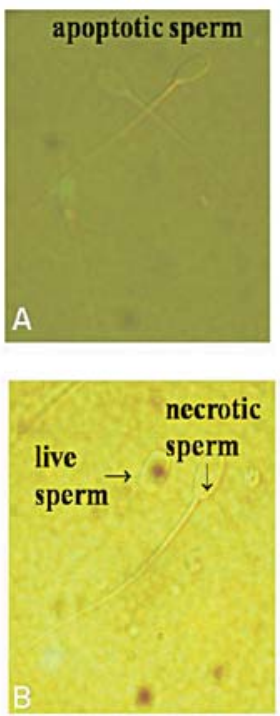
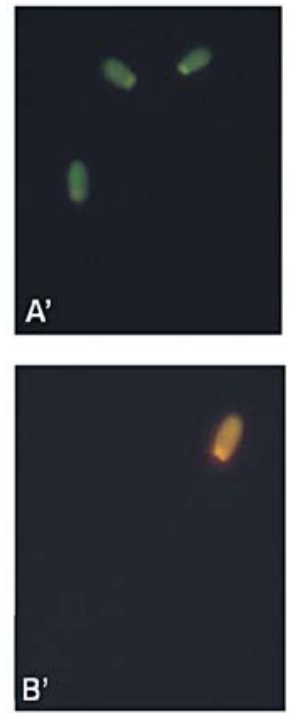
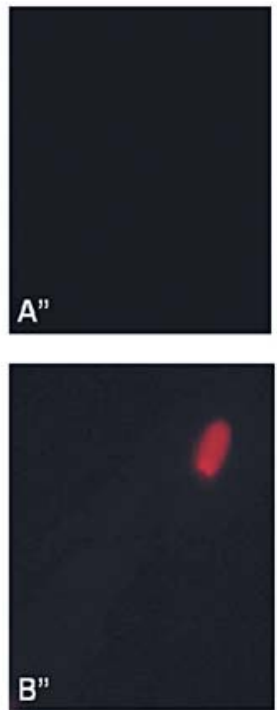

Figure 2. Phase-contrast images (A, B) and fluorescence microscopy (x400) displaying spermatozoa that were fluorescently stained with YO-PRO-1 and PI probes
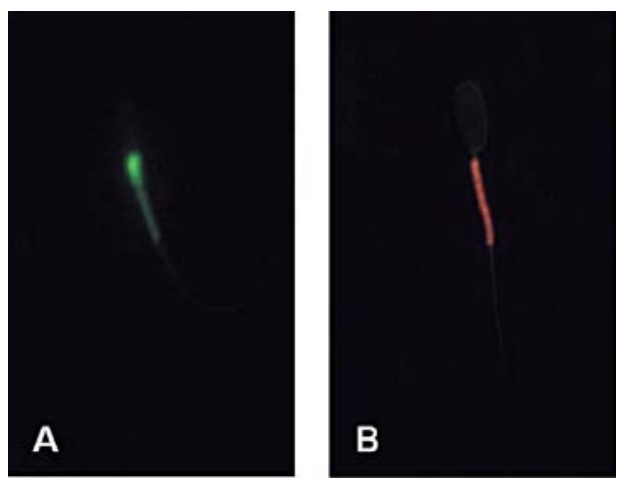

Figure 3. Fluorescence microscopy (x400) displaying spermatozoa that were fluorescently stained with mitochondrial probes. Spermatozoa that were analysed utilizing JC-1 displayed either green mitochondria (A) or red-orange (B) mitochondria 
Correlation coefficients between phenomena analysed are shown in Table 3 . The $\Delta \Psi m$ analysis by JC-1 revealed that mitochondrial function was highly correlated with both sperm motility $(\mathrm{r}=0.936)$ and evaluation for apoptosis/necrosis by $\mathrm{YO}-$ PRO-1/PI assay $(\mathrm{r}=0.960)$.

Table 3. Correlation among sperm motility, mitochondrial activity (JC-1) and membrane integrity (YO-PRO-1/PI) in 5 boars (3 samples/boar); $\mathrm{P}<0.01$

\begin{tabular}{lccc}
\hline \multirow{2}{*}{ Item } & Motility & JC-1 & YO-PRO-1/PI \\
& $\mathrm{r}$ & $\mathrm{r}$ & $\mathrm{r}$ \\
\hline Motility & - & 0.936 & 0.908 \\
JC-1 & 0.936 & - & 0.960 \\
YO-PRO-1/PI & 0.908 & 0.960 & - \\
\hline
\end{tabular}

\section{DISCUSSION}

In the present study, two fluorescence techniques have been used to assess apoptotic and necrotic changes expressed in boar plasma membrane integrity and mitochondrial activity during liquid preservation.

The conventional parameter given most importance has been the motility of sperm in the ejaculate. As expected, a decrease in motility was observed during semen storage, which was also noted by Waterhouse et al. (2004). This estimation is not sufficient for evaluating semen biological value.

YO-PRO-1/PI assay has been proposed as a very sensitive method for detecting apoptotic changes in the sperm plasma membrane (Pena et al., 2005). We observed that sperm emitting green fluorescence are spermatozoa which may show early damage during storage, enabling YO-PRO-1 but not PI. Therefore, the use of a fluorescence probe such as YO-PRO-1, that penetrates cells as they undergo apoptotic changes, at which first steps membranes become slightly permeable, makes YO-PRO-1 an useful tool to detect sperm membrane changes. In general, we observed inconsiderable decreasing of apoptotic sperm during storage, but in the same ejaculates, both increased and decreased numbers of apoptotic spermatozoa were observed during semen storage at $15-17^{\circ} \mathrm{C}$ (data not shown). Necrotic sperm stained both with YO-PRO-1 and PI. YO-PRO-1 penetrates both early and late damaged membranes, producing green fluorescence in sperm nuclei; PI permeates through a damaged membrane and produces red fluorescence. When the plasma membrane loses integrity, PI penetrates to the nucleus and quenches YO-PRO-1 with a consequent change in colour (Wronski, 2002). As storage time increased, the number of necrotic spermatozoa in semen increased. The subpopulation of live cells using this staining showed no fluorescence. As expected, a decrease in this population of sperm was observed during storage time. 
Due to the sensitivity of JC-1 to transient membrane potentials, a decrease in membrane potential has been associated with a decrease in aggregates along with an increase in monomers. Our results show that the percentage of spermatozoa with $\Delta \Psi m$-dysfunctional mitochondria increases and reaches the highest level at the sixth day of semen storage. The $\Delta \Psi m$ analysis by JC-1 revealed that the mitochondrial function is highly correlated with both sperm motility and evaluation for apoptosis/necrosis by YO-PRO-1/PI assay. This indicates that sperm motility is associated with and possibly reliant on healthy mitochondria, and that mitochondrial damage may result in a reduction of sperm movement.

Regardless of the above correlations, the assessment of boar ejaculates on the basis of the sperm motility appears to be an insufficient criterion of semen selection for artificial insemination (AI). Boar ejaculates, which are eliminated solely on the basis of low sperm motility, can still have a high potential for AI due to the low frequency of apoptotic and necrotic spermatozoa. This especially concerns cases of motility that fall to the minimal level. Therefore, evaluation of sperm motility should be supplemented with a detection of apoptosis/necrosis (using YO-PRO-1/PI and JC-1 assays) before the suitability of the ejaculates for AI is finally determined. In addition, success of AI using liquid stored semen will increase, since only the more suitable ejaculates will be selected for this procedure.

Laboratory assessment of sperm quality is an essential procedure for many facets of assisted reproduction. The choice of adequate parameters for reproducible, faster, and more sensitive methods has become increasingly desired because it has become more apparent that standard seminal parameters, such as motility and sperm concentration, are insufficient not only to predict fertility, but even to identify subfertile individuals.

\section{CONCLUSIONS}

In conclusion, this study has demonstrated that methods detecting apoptotic and necrotic changes in boar spermatozoa may be a supplementary criterion for evaluating semen quality. Simultaneous analysis of viability and the mitochondrial status provides a useful and thorough method of assessing functional states of sperm cells. This is of great importance, because fertilization success cannot be attributed to the absolute number of motile and morphologically normal spermatozoa alone. To increase success, it is important that spermatozoa are functionally competent as well. 


\section{REFERENCES}

Anzar M., Liwei He., Buhr M.M., Kroetsch T.G., Pauls K.P., 2002. Sperm apoptosis in fresh and cryopreserved bull semen detected by flow cytometry and its relationship with fertility. Biol. Reprod. 66, 354-360

Barroso G., Morshedi M., Oehninger S., 2000. Analysis of DNA fragmentation, plasma membrane translocation of phosphatidylserine and oxidative stress in human spermatozoa. Hum. Reprod. $15,1338-1344$

Chen Z., Hauser R., Trbovich A.M., Shifren J.L., Dorer D.J., Godfrey-Bailey L., Singh N. P., 2006. The relationship between human semen characteristics and sperm apoptosis: a pilot study. J. Andrology 27, 112-120

Donnelly T.E., O'Connell M., McClure N., Lewis S.E.M., 2000. Differences in nuclear DNA fragmentation and mitochondrial integrity of semen and prepared human spermatozoa. Hum. Reprod. 15, 1552-1571

Huo L.J., Ma X.H., Yang Z.M., 2002. Assessment of sperm viability, mitochondrial activity, capacitation and acrosome intactness in extended boar semen during long-term storage. Theriogenology 58, 1349-1360

Idziorek T., Estaquier J., De Bels F., Ameisen J.C., 1995. YOPRO-1 permits cytofluorometric analysis of programmed cell death (apoptosis) without interfering with cell viability. J. Immunol. Method. 185, 249-258

Lue Y.H., Hikim A.P., Swerdloff R.S., Im P., Taing K.S., Bui T., Leung A., Wang C., 1999. Single exposure to heat induces stage-specific germ cell apoptosis in rats: role of intratesticular testosterone on stage specificity. Endocrinology 140, 1709-1717

Marchetti C., Obert G., Deffosez A., Formstecher P., Marchetti P., 2002. Study of mitochondrial membrane potential, reactive oxygen species, DNA fragmentation and cell viability by flow cytometry in human sperm. Hum. Reprod. 17, 1257-1265

Martin G., Sabido O., Durand P., Levy R., 2004. Cryopreservation induces an apoptosis-like mechanism in bull sperm. Biol. Reprod. 71, 28-37

Oehninger S., Morshedi M., Weng S.L., Taylor S., DuranH., BeebeS., 2003. Presence and significance of somatic cell apoptosis markers in human ejaculated spermatozoa. Reprod. Biomed. Online 7, 469-476

Pena F.J., Saravia F., Johannisson A., Walgren M., Rodriguez-Martinez H., 2005. A new and simple method to evaluate early membrane changes in frozen-thawed boar spermatozoa. Int. J. Androl. $28,107-114$

Sakkas D., Mariethoz E., Manicardi G., Bizzaro D., Bianchi P.G., Bianchi U., 1999. Origin of DNA damage in ejaculated human spermatozoa. Rev. Reprod. 4, 31-37

Sakkas D., Seli E., Manicardi G.C., Nijs M., Ombelet W., Bizzaro D., 2004. The presence of abnormal spermatozoa in the ejaculate: did apoptosis fail? Hum. Fertil. 7, 99-103

Waterhouse K.E., De Angelis P.M., Haugan T., Paulenz H., Hofmo P.O., Farstad W., 2004. Effect of in vitro storage time and semen-extender on membrane quality of boar sperm assessed by flow cytometry. Theriogenology 62, 1638-1651

Wronski R., Golob N., Grygar E., Winsdish M., 2002. Two color, fluorescence-based microplate assay for apoptosis detection. Biotechniques 32, 666-668 